Printed in Great Britain

\title{
THE CHEMISTRY OF ETHYLENEDIAMINE TETRA-ACETIC ACID IN SEA WATER
}

\author{
By C. P. SPENCER \\ Marine Biology Station, University College of North Wales
}

(Text-figs. I-IO)

In recent years, ethylenediamine tetra-acetic acid (EDTA) has been extensively used in culture media for unicellular algae. This, and other chelating agents are also becoming widely used in toxicity studies with plants and animals, in analysis and for controlling the ionic concentration of certain metal ions in a wide variety of studies.

EDTA forms complexes with a large number of metallic cations, and in the presence of sufficient EDTA only very small amounts of many metals can exist as free ions. The use of EDTA as a metal buffer system has been advocated by numerous workers. These metal buffer systems depend for their operation upon the equilibrium between the metal cations, the EDTA and the chelate. Providing that the concentrations of the chelate and the chelating ion are large compared with that of the free metal ion, such a system will tend to maintain a constant concentration of metallic ions, should these be continuously removed by a biological system.

The available data suggest that EDTA is not readily metabolized by most forms of life, and moreover it does not appear to be markedly toxic except by virtue of its metal-chelating reactions. Its use under the correct conditions for controlling metal ion concentrations in biological systems therefore has much to commend it.

The physical chemistry of metal-EDTA equilibria has been examined by several workers (Chaberek, Bersworth \& Martell, I955; Raaflaub, 1956) and methods for calculating the concentrations of free metal ion at equilibrium outlined. These treatments, however, are limited to simple cases when only one chelating metal ion is present. The biologist is inevitably faced with working with systems containing many reacting metal ions (e.g. in sea water). Data regarding the free metal ion concentrations existing in equilibrium with EDTA in such systems is of obvious importance if the use of EDTA is to be fully exploited. This paper records some data on the equilibria set up when EDTA is added to sea water and on the effect of variations of certain controlling factors. 


\section{THE DISSOCIATION OF EDTA}

In distilled water EDTA behaves as a typical quadribasic weak acid.

$$
\begin{aligned}
\mathrm{H}_{4} Y & \rightleftharpoons \mathrm{H}_{3} Y^{-1}+\mathrm{H}^{+} \\
\mathrm{H}_{3} Y^{-1} & \rightleftharpoons \mathrm{H}_{2} Y^{-2}+\mathrm{H}^{+} \\
\mathrm{H}_{2} Y^{-2} & \rightleftharpoons \mathrm{H}^{-3}+\mathrm{H}^{+} \\
\mathrm{H}^{-3} & \rightleftharpoons Y^{-4}+\mathrm{H}^{+}
\end{aligned}
$$

where $Y^{-4}$ represents the fully dissociated EDTA quadrivalent anion. The third and fourth dissociation constants may be determined directly from the titration curve which gives values of $\mathrm{pK}_{3}=6 \cdot \mathrm{I}$ and $\mathrm{pK}_{4}=10 \cdot 2$. The first and second dissociation constants must be determined indirectly and the reported values are $\mathrm{pK}_{1}=\mathrm{I} \cdot 9$ and $\mathrm{pK}_{2}=2 \cdot 6$ respectively (Calvin \& Martell, I953).

When chelation of a metallic ion occurs it results in the displacement of the weakly acidic protons, as for example in the reaction:

$$
M^{2+}+\mathrm{H}_{2} Y^{2-} \rightleftharpoons M Y^{2-}+2 \mathrm{H}^{+}
$$

where $M^{2+}$ represents a divalent metal cation.

Thus, when EDTA is added to sea water, chelation of the metallic ions causes a fall in $\mathrm{pH}$. A typical titration curve for EDTA in sea water is shown in Fig. I (p. 134).

The curve of Fig. I was obtained by titrating sea water of $18.5 \%$ chlorinity containing $9 \times 1 \mathrm{IO}^{-3} \mathrm{M}$ disodium EDTA with $2 \times \mathrm{IO}^{-2} \mathrm{~N}-\mathrm{NaOH}$. The sea water contained 0.0023 equivalent per litre excess base as determined by the method of Mitchell \& Rakestraw (I933). The titration was carried out in a carbon dioxide-free atmosphere. $\mathrm{pH}$ measurements were made by means of a glass electrode. Theoretically, two equivalents of alkali should be required to neutralize the EDTA. The discrepancy between the theoretical and the observed end-point is accounted for by the excess base present in the sea water.

The shape of the titration curve obtained is reminiscent of a strong acidstrong base titration and the effect of additions of EDTA to sea water followed by neutralization with alkali is comparable with the results of adding an equivalent quantity of any strong acid. It should be noticed that, due to the operation of the equilibrium (I), the system acts as a $\mathrm{pH}$ buffer in the region of $\mathrm{pH}_{4}$-5. For most biological purposes, however, the $\mathrm{pH}$ of EDTA-treated sea water will be restored to $\mathrm{pH}_{7}$ or above, and in this region the EDTA system has no buffering capacity and the bicarbonate-carbonate buffering system will therefore remain virtually unaffected. The $\mathrm{pH}$ of EDTA-treated sea water may therefore be adjusted by considering the EDTA to be acting as a strong acid and adjusting the resultant carbonate alkalinity and partial pressure of carbon dioxide as required. 
These considerations apply only when an excess of strongly chelating cations is present. In sea water the concentration is reached at about $6.0 \times 10^{-2} M$ EDTA. At higher EDTA concentrations the system is more complex and the buffer capacity of the sea water will be affected at physiological $\mathrm{pH}$ values.

\section{THEORETICAL TREATMENT OF SYSTEM AND DERIVATION OF FORMULAE}

When EDTA is added to sea water a series of equilibria of the following type operate:

$$
\begin{gathered}
M_{1}^{2+}+Y^{4-} \rightleftharpoons M_{1} Y^{2-} \\
M_{2}^{2+}+Y^{4-} \rightleftharpoons M_{2} Y^{2-} \\
\text { etc. }
\end{gathered}
$$

where $M^{2+}$ represents the divalent chelating cations present. In addition, the four dissociations of EDTA must be considered. At $\mathrm{pH}$ values above about 3-4, the concentrations of $\mathrm{H}_{4} Y$ and $\mathrm{H}_{3} Y^{1-}$ are not appreciable and the first and second dissociations can therefore be neglected for most biological purposes.

For multi-equilibria systems of this type it is possible to write a number of simultaneous equations.

Thus:

$$
\frac{\left[M_{1} Y^{2-}\right]}{\left[M_{1}^{2+}\right]\left[Y^{4-}\right]}=K_{M_{1}}, \frac{\left[M_{2} Y^{2-}\right]}{\left[M_{2}^{2+}\right]\left[Y^{4-}\right]}=K_{M_{2}}, \quad \text { etc., }
$$

where the quantities in brackets represent concentrations, and by rewriting the analogous equations for the third and fourth dissociations of EDTA in the form

$$
\frac{\left[\mathrm{H} Y^{3-}\right]}{\left[\mathrm{H}^{+}\right]\left[Y^{4-}\right]}=K_{A} \text { and } \frac{\left[\mathrm{H}_{2} Y^{2-}\right]}{\left[\mathrm{H}^{+}\right]^{2}\left[Y^{4-}\right]}=K_{B},
$$

where $K_{A}=\mathrm{I} / K_{4}$ and $K_{B}=\mathrm{I} / K_{3} K_{4}, K_{3}$ and $K_{4}$ being the dissociation constants for the third and fourth dissociations of EDTA. In addition we have the mass equations:

$$
\begin{aligned}
{\left[\operatorname{Total} M_{1}\right]=\left[M_{1}^{2+}\right]+\left[M_{1} Y^{2-}\right] } \\
{\left[\text { Total } M_{2}\right]=\left[M_{2}^{2+}\right]+\left[M_{2} Y^{2-}\right] } \\
\text { etc. }
\end{aligned}
$$

and $[$ Total $Y]=\left[M_{1} Y^{2-}\right]+\left[M_{2} Y^{2-}\right]+$ etc. $+\left[\mathrm{H}_{2} Y^{2-}\right]+\left[\mathrm{H} Y^{3-}\right]+\left[Y^{4-}\right]$.

In general if a system consisting of $n$ competing ions is considered it is possible to set up $(2 n+\mathrm{I})$ simultaneous equations. Providing the values of the stability 
constants ( $K_{M_{1}}, K_{M_{\mathrm{a}}}$, etc.) of the metal chelates, the values of the dissociation constants of EDTA $\left(K_{3}\right.$ and $\left.K_{4}\right)$ and the total concentrations of EDTA (Total $Y$ ) and of the chelating cations (Total $M_{1}$, Total $M_{2}$, etc.) are known, then the system of equations contains $(2 n+\mathrm{I})$ unknowns (i.e. $M_{1}^{2+}, M_{1} Y^{2-}$, $M_{2}^{2+}$, etc.) and may be solved for any of these.

When the case of EDTA in sea water is considered the value of $n$ is large and the system of simultaneous equations becomes very cumbersome to solve by direct means. The following alternative treatment was therefore used.

Let $\frac{\left[M_{1}^{2+}\right]}{\left[Y^{4-}\right]}=a$, then $\left[M_{1}^{2+}\right]=a\left[Y^{4-}\right]$.

Similarly $\left[\mathrm{M}_{2}^{2+}\right]=b\left[\mathrm{Y}^{4-}\right]$,

etc.

Also let $\frac{\left[M_{1} Y^{2-}\right]}{\left[Y^{4-}\right]}=\alpha$, then $\left[M_{1} Y^{2-}\right]=\alpha\left[Y^{4-}\right]$.

And let $\frac{\left[\mathrm{H}_{3} Y^{3-}\right]}{\left[Y^{4-}\right]}=\lambda$, Similarly $\left[M_{2} Y^{2-}\right]=\beta\left[Y^{4-}\right]$.

And let $\frac{\mathrm{I}}{\left[Y^{4-}\right]}=f$. then $\left[H Y^{3-}\right]=\lambda\left[Y^{4-}\right]$, and $\left[\mathrm{H}_{2} Y^{2-}\right]=\mu\left[Y^{4-}\right]$.

Then from the equations for the stability constants of the metal chelates and for the dissociation constants of EDTA

$$
K_{M_{1}}=f(\alpha / a), \quad K_{M_{\mathrm{a}}}=f(\beta / b), \quad \text { etc. }
$$

Also

$$
K_{A}\left[\mathrm{H}^{+}\right]=\lambda \text { and } K_{B}\left[\mathrm{H}^{+}\right]^{2}=\mu \text {. }
$$

Now

$$
\text { [Total } \left.M_{1}\right]=\left[M_{1}^{2+}\right]+\left[M_{1} Y^{2-}\right]
$$$$
=a\left[Y^{4-}\right]+\alpha\left[Y^{4-}\right] .
$$

$f\left[\right.$ Total $\left.M_{1}\right]=a+\alpha$

$$
\begin{gathered}
=\alpha+f \alpha / K_{M_{1}} \\
\alpha=\frac{f\left[\text { Total } M_{1}\right]}{\mathrm{I}+f / K_{M_{1}}}
\end{gathered}
$$

Similarly

$$
\beta=\frac{f\left[\text { Total } M_{2}\right]}{\mathrm{I}+f / K_{M_{2}}},
$$

etc. 
Now

$[$ Total $Y]=\left[M_{1} Y^{2-}\right]+\left[M_{2} Y^{2-}\right]+$ etc. $+\left[\mathrm{H}_{2} Y^{2-}\right]+\left[\mathrm{H} Y^{3-}\right]+\left[Y^{4-}\right]$ $=\alpha\left[Y^{4-}\right]+\beta\left[Y^{4-}\right]+$ etc. $+\mu\left[Y^{4-}\right]+\lambda\left[Y^{4-}\right]+\left[Y^{4-}\right]$.

$f[$ Total $Y] \alpha+\beta+$ etc. $+\mu+\lambda+\mathbf{I}$.

$f[$ Total $Y]=\frac{f\left[\text { Total } M_{1}\right]}{\mathrm{I}+f / K_{M_{1}}}+\frac{f\left[\text { Total } M_{2}\right]}{\mathrm{I}+f / K_{M_{2}}}+$ etc. $+K_{A}\left[\mathrm{H}^{+}\right]+K_{B}\left[\mathrm{H}^{+}\right]^{2}+\mathbf{I}$.

This equation may be solved for $f$. It will be seen that the function $f$ is by definition the reciprocal of the concentration of the fully dissociated form of EDTA (i.e. $Y^{4-}$ ). In a system consisting of several cations each competing for the $Y^{4-}$ anion, it is the concentration of this ion, together with the total amount of a particular metal species present and the appropriate stability constant, which determines the equilibrium concentration of the unchelated metal ions. All the chelating cations present will affect to a greater or less extent the value of $f$ for a certain concentration of EDTA. When the general equation has been solved for $f$ for a particular case the concentration of $Y^{4-}$ can be calculated. Substitutions of this value in the equation

$$
\left[M^{2+}\right]=\frac{[\text { Total } M]}{K_{M}\left[Y^{4-}\right]+\mathrm{I}}
$$

together with the appropriate values of [Total $M]$ and $K_{M}$ allows the calculation of $\left[M^{2+}\right]$.

The general equation for $f$ is only directly applicable to a system of any number of competing metallic ions which react with $Y^{4-}$ to give chelates of the type $M Y^{2-}$. If chelates of other structure are found the form of the terms in the general equation corresponding to these chelates alters. For such chelates it is in general a simple matter to recast the equilibrium constant equations so that they can be incorporated in the general formula.

As an example we may consider the case of calcium. This forms chelates with both the $Y^{4-}$ and $\mathrm{HY}^{3-}$ anions of EDTA according to the following reactions (Calvin \& Martell, 1953):

$$
\begin{aligned}
\mathrm{Ca}^{2+}+Y^{4-} & \rightleftharpoons \mathrm{Ca} Y^{2-} \\
\mathrm{Ca}^{2+}+\mathrm{HY}^{3-} & \rightleftharpoons \mathrm{CaH} Y^{1-} .
\end{aligned}
$$

Hence $\frac{\left[\mathrm{CaY} Y^{2-}\right]}{\left[\mathrm{Ca}^{2+}\right]\left[Y^{4-}\right]}=K_{1}$ and $\frac{\left[\mathrm{CaH} Y^{1-}\right]}{\left[\mathrm{Ca}^{2+}\right]\left[\mathrm{HY}^{3-}\right]}=K_{2}$.

The stability constants for both reactions are known and from these and the appropriate proton association constant of EDTA

$$
K_{A}=\frac{\left[\mathrm{H} Y^{3-}\right]}{\left[\mathrm{H}^{+}\right]\left[Y^{4-}\right]}
$$


may be derived a fourth constant $\mathrm{K}^{\prime}$ :

$$
K^{\prime}=\frac{\left[\mathrm{CaH} Y^{1-}\right]}{\left[\mathrm{Ca}^{2+}\right]\left[Y^{4-}\right]\left[\mathrm{H}^{+}\right]} \text {where } K^{\prime}=K_{2} \times K_{A} .
$$

The equations for $K_{1}$ and $K^{\prime}$ are now in a suitable form for manipulation by the procedure used in deriving the general formula. It can be shown that this substitution results in two terms

$$
\frac{f[\text { Total Ca }] K_{1}}{f+K_{1}+K^{\prime}\left[\mathrm{H}^{+}\right]} \text {and } \frac{f[\text { Total Ca }] K^{\prime}\left[\mathrm{H}^{+}\right]}{f+K_{1}+K^{\prime}\left[\mathrm{H}^{+}\right]}
$$

in place of the usual single term of the form $\frac{f[\text { Total } M]}{\mathrm{I}+f / K_{M}}$ as in the case of ions forming only one chelate.

Insertion of these terms in the general formula and solving for $f$ enables each term to be evaluated and hence the concentration of $\mathrm{Ca}^{2+}, \mathrm{CaY}^{2-}$ and $\mathrm{CaHY}{ }^{1-}$ calculated for a specific case. Calculation shows that in sea-water solutions of EDTA, formation of the $\mathrm{CaHY} Y^{1-}$ chelate is negligible at $\mathrm{pH}$ values of 5 and above. At $\mathrm{pH}_{4}$ the influence of $\mathrm{CaHY}^{-1}$ formation on the value of $f$ is only slight and for most purposes can be neglected. This approximation is only valid if the concentration of $\mathrm{HY}^{3-}$ is kept low by the presence of a stoichiometric excess of strongly chelated cations. If appreciable formation of $\mathrm{CaHY} Y^{-1}$ does occur it results in a reduction in the concentration of $\mathrm{Ca}^{2+}$.

The general formula includes terms to allow for the effect of the third and fourth dissociation constants of EDTA. If systems at $\mathrm{pH}$ values below 4 are to be considered the first and second dissociations will become important. In such cases the $\mathrm{pH}$-dependent terms in the general formula must be extended to include the terms $+K_{C}\left[\mathrm{H}^{+}\right]^{3}$ and $+K_{D}\left[\mathrm{H}^{+}\right]^{4}$, where

$$
K_{C}=\frac{I}{K_{2} K_{3} K_{4}} \text { and } K_{D}=\frac{\mathrm{I}}{K_{1} K_{2} K_{3} K_{4}}
$$

\section{APPLICATION OF THE GENERAL FORMULA TO SEA WATER CONTAINING EDTA}

The constants quoted in Table I are the apparent stability constants determined in solutions of known ionic strength $(\mu=0 \cdot I)$. These constants are related to the thermodynamic dissociation constants $K$ by the relationship

$$
K=K^{\prime} \frac{\gamma M Y^{2-}}{\gamma M^{2+} \times \gamma Y^{4-}},
$$

where $K^{\prime}$ is the apparent dissociation constant and $\gamma M Y^{2-}, \gamma M^{2+}$ and $\gamma Y^{4-}$ are the activity coefficients of the metal-EDTA anion, the metal cation and the fully dissociated quadrivalent EDTA anion respectively. 
Due to the nature of the ions relatively large variations in the activity coefficients and the apparent value of $K$ with ionic strength can be expected. The reported constants are not therefore strictly applicable to the equilibria set up in EDTA solution in sea water. There is insufficient data for a rigorous treatment to assess the variations in the individual apparent constants with variations of ionic strength. It seems justifiable, however, to attempt an approximate treatment on the basis of the data available.

TABLE 1. STABILITY CONSTANTS OF THE EDTA CHELATES OF SOME BIOLOGICALLY IMPORTANT IONS (CALVIN \& MARTELL, I953)

$\begin{array}{lrll}\mathrm{Na}^{+} & \mathrm{I} \cdot 66 & \mathrm{Mn}^{2+} & \mathrm{I} 3.4 \\ \mathrm{Sr}^{2+} & 8.36 & \mathrm{Co}^{2+} & \mathrm{I} 6 \cdot \mathrm{I} \\ \mathrm{Mg}^{2+} & 8 \cdot 69 & \mathrm{Zn}^{2+} & \mathrm{I} 6 \cdot \mathrm{I} \\ \mathrm{Ca}^{2+} & 10.59 & \mathrm{Cu}^{2+} & \mathrm{I} 8.3 \\ \mathrm{Ca}^{2+} & 3.5 \mathrm{I}^{\star} & & \end{array}$

Unless otherwise stated the values given are the $\log K$ values for the $M Y^{2-}$ chelate.

$$
{ }^{\star} K=\frac{\mathrm{CaH} Y^{-1}}{\mathrm{Ca}^{2+} \mathrm{H} Y^{-3}} \text {. }
$$

Carini \& Martell (1952) have investigated the changes with ionic strength of the apparent stability constant of the calcium-EDTA chelate. Their results show that in sea water of $19 \% \mathrm{Cl}$ the apparent stability constant will be reduced by about a power of Io compared with its value at $\mu=0 \cdot \mathrm{I}$. Variations will also be caused in the values of the apparent dissociation constants of EDTA, but these can be neglected for the present approximation.

It seems reasonable to suppose that the variation in the activity coefficients $\gamma M Y^{2-}, \gamma M^{2+}$ with changes in ionic strength will be of the same order for all divalent metallic cations and as an approximation the apparent stability constants to be used in sea-water solutions can therefore be reduced by a similar factor. This approximation can only be justified in the case of equilibria involving divalent cations and the quadrivalent EDTA anion. As will be seen from what follows below, this limitation still allows the calculation of some data of value.

The reacting cations present in sea water fall into two groups. On the one hand there are the major constituents, $\mathrm{Na}^{+}, \mathrm{Mg}^{2+}$, and $\mathrm{Ca}^{2+}$ which are present in concentrations of the order of $10^{-2} \mathrm{M}$ or above, and on the other the remaining cations present in micro- or submicromolar concentrations. Application of the general formula in the specific case of the addition of a certain concentration of EDTA to sea water indicates that for any particular case the value of $f$ is largely controlled by a relatively small number of the chelating ions present. The dominance of an ion in this role is determined by its concentration relative to the concentration of EDTA and by the value of the stability constant of its chelate. It is thus possible to ignore the effects of many of the ions when calculating the value of $f$ for a certain concentration of EDTA. With EDTA concentrations between $\mathrm{IO}^{-2}$ and $1 \mathrm{IO}^{-6} \mathrm{M}$ the influence of 
the chelating reactions involving the cations of magnesium and calcium are predominant. In the case of calcium only the formation of the $\mathrm{Ca} Y^{2-}$ chelate is important, since the term corresponding to the formation of $\mathrm{CaH} Y^{3-}$ has little effect on the value of $f$.

Fig. 2 shows the variation in the value of $f$ when various quantities of EDTA are added to sea water at $\mathrm{pH} 8.0$. The values of $f$ plotted in this curve were

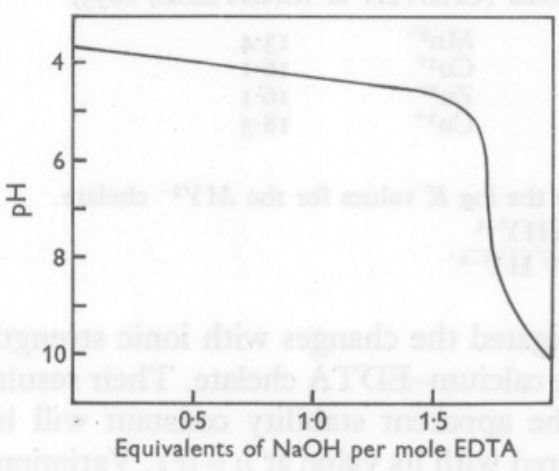

Fig. I

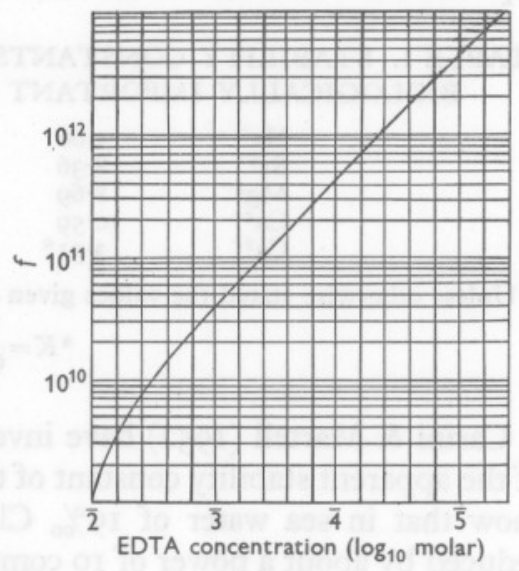

Fig. 2

Fig. I. Titration curve for a solution of EDTA in sea water.

Fig. 2. Variation of $f$ with the concentration of EDTA in sea water of $19 \% \mathrm{Cl}$ at $\mathrm{pH} 8 \cdot 0$.

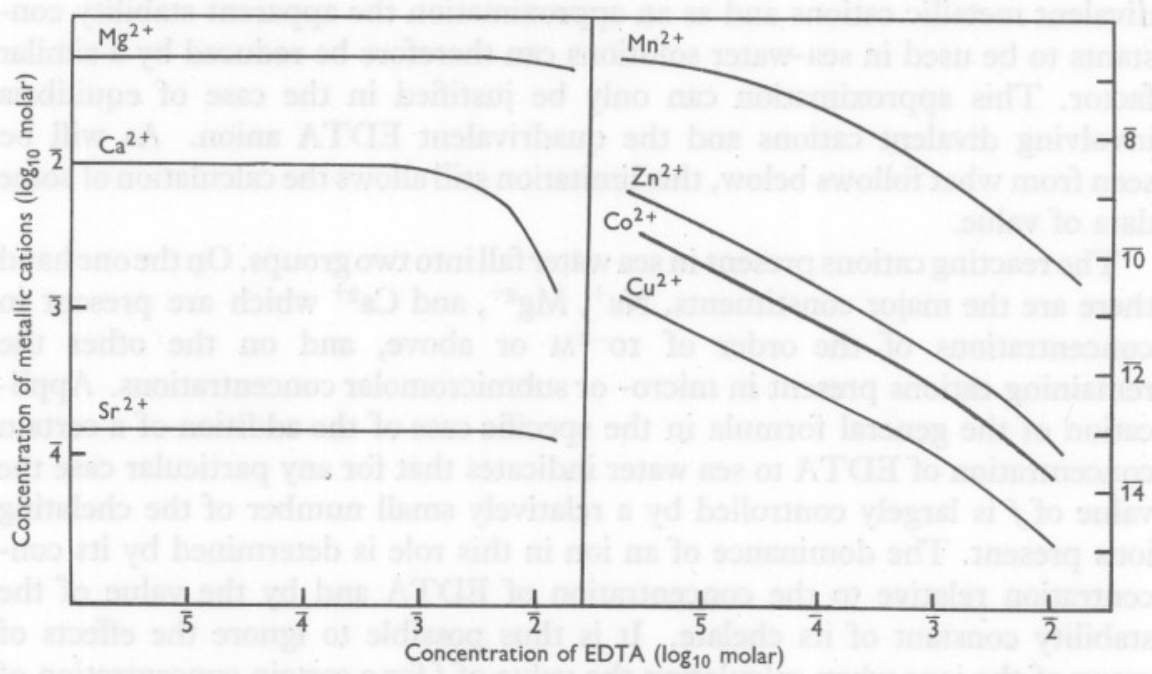

Fig. 3. Variation of the concentration of certain metallic cations in equilibrium with various concentrations of EDTA in sea water of $19 \% \mathrm{Cl}$ at $\mathrm{pH} 8.0$. 
obtained by applying the general formula to solutions of EDTA in sea water of $19 \%$ chlorinity and cationic composition reported as typical by Sverdrup, Johnson \& Fleming (1942). As a first approximation the influence on the value of $f$ of all cations other than magnesium and calcium was neglected.

Values of $f$ taken from the curve are of sufficient accuracy for many purposes. If greater accuracy is required the curve may be used for obtaining an approximate root of the equation which may then be further refined to the desired degree.

Using the value of $f$ obtained from the curve in Fig. I the concentrations at equilibrium of the unchelated portions of several biologically important cations have been calculated for a range of EDTA concentrations. The results are shown in Fig. 3. The total concentrations of the various cations used in these calculations are taken from the same authority as the data for the construction of Fig. 2.

An examination of the ultra-violet absorption spectrum of appropriate mixtures of calcium and magnesium ions in the presence of EDTA provides some experimental verification of the theoretical results set out in Fig. 3 above. EDTA itself absorbs strongly below about $260 \mathrm{~m} \mu$. Chelation with calcium or magnesium causes a shift of the absorption to shorter wavelengths. The absorption maxima cannot be determined at the concentrations which have been used and precise measurements are thus not possible. However, the curves

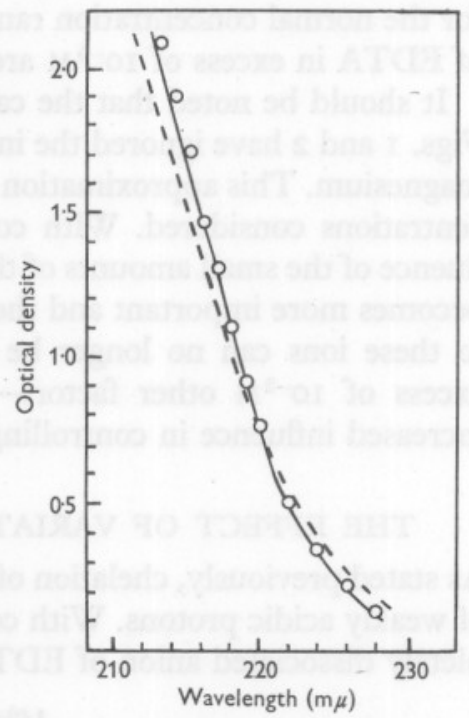

Fig. 4. Absorption spectra of $5 \times 10^{-3}$ EDTA in $M / 15$ phosphate buffer at $\mathrm{pH} 7.0$ with additions of: (a) $5 \times \mathrm{IO}^{-3} \mathrm{M}-$ $\mathrm{Ca}^{2+}$, ; (b) $5 \times \mathrm{IO}^{-2} \mathrm{M}^{2} \mathrm{Mg}^{2+}$, $----;$ and (c) $5 \times \mathrm{IO}^{-3} \mathrm{M}-\mathrm{Ca}^{2+}+$ $5 \times \mathrm{IO}^{-2} \mathrm{M}-\mathrm{Mg}^{2+}, \mathrm{O} O \mathrm{O}$. Absorption measured in I cm cuvette against phosphate buffer as blank using a Unicam SP 500 spectrophotometer. obtained when either the calcium or magnesium chelate is used are separated (Fig. 4). If mixtures of calcium and magnesium ions in the presence of EDTA are examined, at relative concentrations such that the calcium is in stoichiometric excess of the EDTA, it is seen that the absorption spectrum of the mixture approximates to that of the calcium chelate, indicating that little magnesium is chelated under these conditions. This is as predicted by the theoretical treatment. If the concentration of EDTA is increased until it approaches the stoichiometric amount required by the calcium the absorption spectrum of the mixture starts to deviate slightly from that of the pure calcium chelate. Marked deviation, indicating appreciable formation of the magnesium 
chelate does not, however, occur until the EDTA concentration is equal to or exceeds the concentration equivalent to the calcium.

The case of iron has so far been omitted from the discussion and no data for the concentration of either ferrous or ferric ions which can exist in equilibrium with various concentrations of EDTA in sea water is given in Fig. 3. For several reasons the case of iron is more complicated and it will be discussed elsewhere. For most purposes, however, it is safe to assume that the presence of iron will have little effect upon the other equilibria and this is certainly true for the normal concentration range of iron found in sea water when additions of EDTA in excess of $10^{-5} \mathrm{M}$ are used.

It should be noted that the calculations involved in obtaining the data for Figs. I and 2 have ignored the influence of all cations, other than calcium and magnesium. This approximation is reasonable within the range of EDTA concentrations considered. With concentrations of the order of $10^{-6} \mathrm{M}$ the influence of the small amounts of the more strongly chelated ions such as copper becomes more important and the terms in the general formula corresponding to these ions can no longer be ignored. With concentrations of EDTA in excess of $\mathrm{IO}^{-2} \mathrm{M}$ other factors-especially the $\mathrm{pH}$ of the system-have an increased influence in controlling the value of $f$.

\section{THE EFFECT OF VARIATIONS OF PH ON THE EQUILIBRIA}

As stated previously, chelation of cations by EDTA involves the displacement of weakly acidic protons. With certain exceptions chelation involves the completely dissociated anion of EDTA

$$
\mathrm{M}^{2+}+\mathrm{Y}^{4-} \rightleftharpoons \mathrm{MY} \mathrm{Y}^{2-}
$$

and the tendency for hydrogen ions to associate with $Y^{4-}$

$$
\mathrm{H}^{+}+Y^{4-} \rightleftharpoons \mathrm{HY}^{3-}
$$

may thus be considered to be direct competition with metallic cation. The second proton association

$$
\mathrm{H}^{+}+\mathrm{H} Y^{3-} \rightleftharpoons \mathrm{H} Y^{2-}
$$

will compete indirectly. As before, the effect of the third and fourth proton association can be neglected when considering systems at $\mathrm{pH}$ values above $4^{\circ} \mathrm{O}$.

Fig. 5 shows the variations with $\mathrm{pH}$ of the sum of the two $\mathrm{pH}$-dependent terms in the general formula. $K_{A}\left[\mathrm{H}^{+}\right]+K_{B}\left[\mathrm{H}^{+}\right]^{2}$ increases rapidly with falling $\mathrm{pH}$, reaching values of the order of $\mathrm{IO}^{6}$ at $\mathrm{pH}$. When the general formula is applied to $19 \%$ sea-water solutions of EDTA, the terms corresponding to the ions of magnesium and calcium, which are of dominant influence in controlling the value of $f$, are of the order of $10^{7}$ and above. It therefore follows that at $\mathrm{pH}$ values of 6 and above the $\mathrm{pH}$ will have little effect on the equilibria set up when EDTA is added to sea water. Below a pH 
of 6 the two $\mathrm{pH}$-dependent terms will increasingly affect the value of $f$ and hence the equilibrium concentration of the unchelated metallic cations.

The values of the first and second proton association constants are such as to suggest at first sight that the more stable first proton association might be expected to have a marked effect, especially since the value of the association constant for this proton is of the same order as the formation constant of the

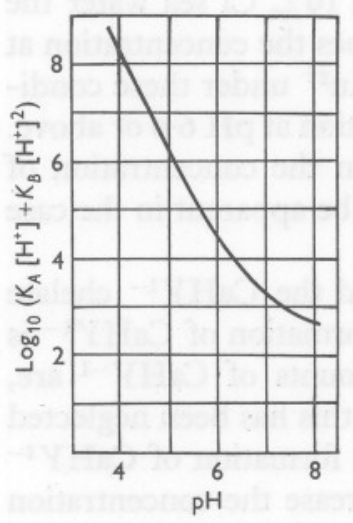

Fig. 5

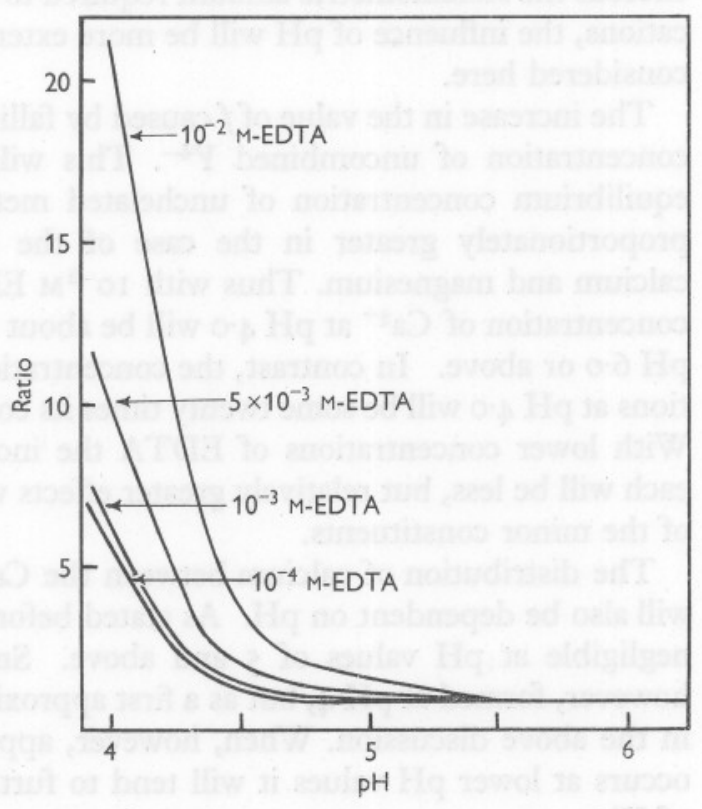

Fig. 6

Fig. 5. Variations with $\mathrm{pH}$ of the two $\mathrm{pH}$-dependent terms in the general formula. Vertical axis shows value of $\log _{10}\left(K_{A}\left[\mathrm{H}^{+}\right]+K_{B}\left[\mathrm{H}^{+}\right]^{2}\right)$

Fig. 6. Ratio of $f$ at various $\mathrm{pH}$ values to $f$ at $\mathrm{pH} 8.0$ in sea water of $19 \% \mathrm{Cl}$ with various concentrations of EDTA.

calcium chelate and $1 \mathrm{I}^{2}$ greater than that of the magnesium chelate. In fact, over the $\mathrm{pH}$ range 8-I0 when the effect of the first proton association might be expected to show, the $\mathrm{pH}$ has little effect on the value of $f$ and hence on the position of the equilibria. Presumably in this $\mathrm{pH}$ range the mass effect of the hydrogen ions is so low compared with the $10^{8}$ times greater amounts of calcium and magnesium that this proton association is of negligible influence in controlling the concentration of $Y^{4-}$ compared with the chelation of calcium and magnesium.

As can be seen from Fig. 6 the relative effect of $\mathrm{pH}$ on the value of $f$ varies with the concentration of EDTA added to the sea water. The effect is apparent at higher $\mathrm{pH}$ values and is of greater magnitude at the lower $\mathrm{pH}$ values, the 
more nearly the concentration of EDTA approaches the total concentration of strongly chelated cations present. With $19 \% \mathrm{Cl}$ sea water this represents about $6 \times \mathrm{IO}^{-2} \mathrm{M}$ EDTA. Additions of EDTA to $19 \% \mathrm{Cl}$ sea water are in any case limited to about this concentration by solubility considerations. It is, however, possible that EDTA may be used in diluted sea water and in such cases it should be noticed that if the concentration of EDTA is equal to or exceeds the stoichiometric amount required to chelate all the strongly chelated cations, the influence of $\mathrm{pH}$ will be more extensive than under the conditions considered here.

The increase in the value of $f$ caused by falling $\mathrm{pH}$ reflects a decrease in the concentration of uncombined $Y^{4-}$. This will result in an increase in the equilibrium concentration of unchelated metal cations, the increase being proportionately greater in the case of the micro-constituents than with calcium and magnesium. Thus with $10^{-2} \mathrm{M}$ EDTA in $19 \% \mathrm{Cl}$ sea water the concentration of $\mathrm{Ca}^{2+}$ at $\mathrm{pH} 4.0$ will be about four times the concentration at $\mathrm{pH} 6.0$ or above. In contrast, the concentration of $\mathrm{Mn}^{2+}$ under these conditions at $\mathrm{pH}_{4} .0$ will be some twenty times its concentration at $\mathrm{pH} 6.0$ or above. With lower concentrations of EDTA the increases in the concentration of each will be less, but relatively greater effects will still be apparent in the case of the minor constituents.

The distribution of calcium between the $\mathrm{Ca} Y^{2-}$ and the $\mathrm{CaH} Y^{1-}$ chelate will also be dependent on $\mathrm{pH}$. As stated before, the formation of $\mathrm{CaH} Y^{1-}$ is negligible at $\mathrm{pH}$ values of 5 and above. Small amounts of $\mathrm{CaH} Y^{-1}$ are, however, formed at $\mathrm{pH}_{4}$, but as a first approximation this has been neglected in the above discussion. When, however, appreciable formation of $\mathrm{CaHY} Y^{1-}$ occurs at lower $\mathrm{pH}$ values it will tend to further decrease the concentration of $\mathrm{Y}^{4-}$.

\section{THE EFFECTS OF VARIATIONS IN THE SALINITY ON THE EQUILIBRIA}

Variations in the salinity of the sea water to which additions of EDTA are made will affect the equilibria due to: $(a)$ variations in the ionic strength of the medium, and $(b)$ variations in the total concentrations of the chelating cations.

Fig. 7 shows the relative variations of $f$ with three concentrations of EDTA in sea water of various salinities. As the salinity falls the decreasing ionic strength is associated with a rise in the apparent stability constants of the chelates and a concomitant increase in chelation. At the same time the total concentration of the chelating cations decreases, with the result that the EDTA is present in proportionately greater quantities.

As can be seen from the appropriate curves in Fig. 7 the relative importance of these two effects depends upon the EDTA concentration. With EDTA concentrations of $\mathrm{IO}^{-2} \mathrm{M}$ decreasing salinity causes an immediate fall in the 
value of $f$, the influence of decreasing ionic strength being masked by the mass effect. With lower concentrations of EDTA, however, falling salinity is at first accompanied by an increase in the value of $f$ due to the lowering of the ionic strength. When the total concentration of chelated cations falls below about 6 times the concentration of EDTA, the mass effect becomes dominant and the value of $f$ falls sharply.

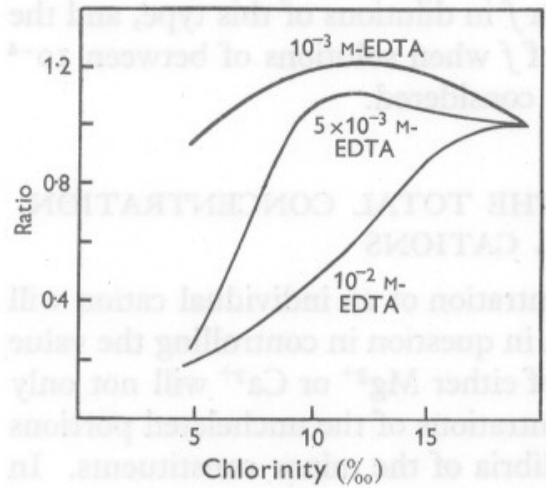

Fig. 7

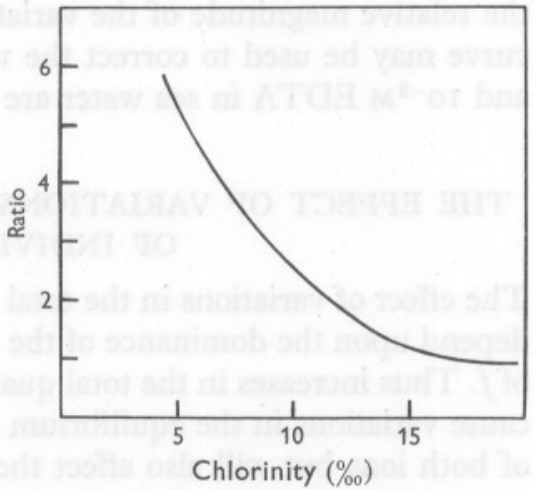

Fig. 8

Fig. 7. Ratio of $f$ in sea waters of various chlorinities to $f$ at $19 \%$ chlorinity with various concentrations of EDTA.

Fig. 8. Ratio of $f$ at various dilutions of a $10^{-3} \mathrm{M}$ solution of EDTA in sea water of $19 \%$ $\mathrm{Cl}$ at $\mathrm{pH} 8.0$.

Both the increase in the value of $f$ due to decreased ionic strength and the decrease in $f$ caused by the mass effect are accompanied by a decrease in the concentration of unchelated metallic cations. The rise in the value of $f$ between I9 and $10 \% \mathrm{Cl}$ with $5 \times \mathrm{IO}^{-3} \mathrm{M}$ EDTA, for example, is a reflexion of the rising stability constants which are paralleled by increasing chelation. Similarly, with the same strength of EDTA below $10 \% \mathrm{Cl}$, the rapidly falling value of $f$ due to the mass effect is accompanied by a further decrease in metallic cation concentration, since the total concentration of the metals is decreasing.

The fall in metallic cation concentration is only slight, due to the effect of the ionic strength, but more marked due to the mass effect. The combined effect is proportionately greater with the minor constituents than with such ions as calcium and magnesium. For example, with additions of $5 \times 10^{-3} \mathrm{M}$ EDTA to sea water of $19 \% \mathrm{Cl}$ the resulting concentrations of $\mathrm{Ca}^{2+}$ and $\mathrm{Cu}^{2+}$ are about $5.5 \times 10^{-3} \mathrm{M}$ and $4.9 \times 10^{-15} \mathrm{M}$ respectively. If the same addition is made to $15 \% \mathrm{Cl}$ sea water the concentrations become $3.53 \times 10^{-3} \mathrm{M}$ and $2.6 \times \mathrm{IO}^{-15} \mathrm{M}$. With sea water of $5 \% \mathrm{Cl}$, however, the concentration of $\mathrm{Ca}^{2+}$ will fall to $\mathrm{I} \cdot 3 \times \mathrm{IO}^{-4} \mathrm{M}$ and that of $\mathrm{Cu}^{2+}$ to about $5 \cdot 8 \times \mathrm{IO}^{-17} \mathrm{M}$.

It may sometimes happen that sea water containing EDTA is diluted with 
distilled water. In this case not only is the ionic strength of the medium reduced and the total concentration of metallic cation, but also the total concentration of EDTA is proportionately reduced. Fig. 8 shows the variation in the value of $f$ when sea water of $19 \% \mathrm{Cl}$ containing $10^{-3} \mathrm{M}$ EDTA is diluted. The proportionate decrease in the total amount of EDTA with decreases in the concentration of the other constituents causes a progressive increase in the value of $f$. The concentration of EDTA has little effect upon the relative magnitude of the variations in $f$ in dilutions of this type, and the curve may be used to correct the value of $f$ when solutions of between Io $^{-4}$ and $10^{-2} M$ EDTA in sea water are being considered.

\section{THE EFFECT OF VARIATIONS IN THE TOTAL CONCENTRATION OF INDIVIDUAL CATIONS}

The effect of variations in the total concentration of an individual cation will depend upon the dominance of the cation in question in controlling the value of $f$. Thus increases in the total quantity of either $\mathrm{Mg}^{2+}$ or $\mathrm{Ca}^{2+}$ will not only cause variations in the equilibrium concentrations of the unchelated portions of both ions but will also affect the equilibria of the minor constituents. In contrast, a small increase in, for example, the total amount of copper present, will have only a negligible effect on the value of $f$, and only the copper equilibrium will thus be affected. If, however, the total amount of copper is increased to a point where the appropriate term in the general formula is no longer of negligible influence on the value of $f$, then further increases in the total copper present will affect not only the $\mathrm{Cu}^{2+}$ equilibrium but also all the other equilibria.

The effects of increasing the total concentration of calcium in a $10^{-3} \mathrm{M}$ solution of EDTA in $19 \% \mathrm{Cl}$ sea water at $\mathrm{pH} 8.0$ will be considered first. As the total amount of calcium present is increased the value of $f$ increases. As shown in Fig. 9, $f$ is directly proportional to the total concentration of calcium for a particular concentration of EDTA. The value of the proportionality constant is greater the more nearly the concentration of EDTA approaches the total concentration of strongly chelated cations. In $19 \% \mathrm{Cl}$ sea water, decreases in the concentration of EDTA below $\mathrm{IO}^{-3} \mathrm{M}$ have little further effect on this proportionality constant.

The increase in $f$ caused by increased total amounts of calcium reflects increases in the equilibrium concentrations of all the strongly chelated cations. Table 2 gives the equilibrium concentration of several cations in a $10^{-3} \mathrm{M}$ solution of EDTA in $19 \% \mathrm{Cl}$ sea water at $\mathrm{pH} 8.0$ appropriate to various total amounts of calcium. The concentrations of free cations increase linearly with increasing total amounts of calcium, the relative increase in each cation being approximately equal, with a given concentration of EDTA, for all cations regardless of the amount present or the value of their respective stability 
constants. The greater increases in $f$ with higher concentrations of EDTA reflect greater increases in the equilibrium concentration of each metallic cation. Nevertheless, even with $10^{-2} \mathrm{M}$ solutions of EDTA, the relative increase in free cations caused by addition of calcium remains approximately the same for a considerable range of calcium additions.

TABLE 2. EFFECT OF INCREASING TOTAL AMOUNT OF CALCIUM IN $10^{-3} \mathrm{M}$ SOLUTIONS OF EDTA IN $19 \%$ CL SEA WATER AT pH 8.0

Total

Ca present (molar)

$\mathrm{I} \cdot \mathrm{O} \times \mathrm{IO}^{-2}$

$2.0 \times 10^{-2}$

$5.0 \times 10^{-2}$

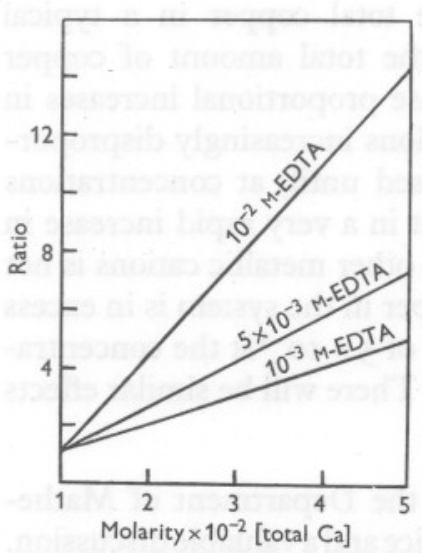

Fig. 9

\begin{tabular}{c|cc}
\multicolumn{3}{c}{ Molar equilibrium concentrations } \\
$\mathrm{Ca}^{2+}$ & $\mathrm{Mn}^{2+}$ & $\mathrm{Cu}^{2+}$ \\
$9.8 \times \mathrm{IO}^{-3}$ & $3.0 \times \mathrm{IO}^{-9}$ & $3.9 \times \mathrm{IO}^{-14}$ \\
$\mathrm{I} \cdot 9 \times \mathrm{IO}^{-2}$ & $6.0 \times \mathrm{IO}^{-9}$ & $7 \cdot 8 \times \mathrm{IO}^{-14}$ \\
$4.9 \times \mathrm{IO}^{-2}$ & $\mathrm{I} \cdot 5 \times \mathrm{IO}^{-8}$ & $2 \cdot 0 \times 10^{-13}$
\end{tabular}

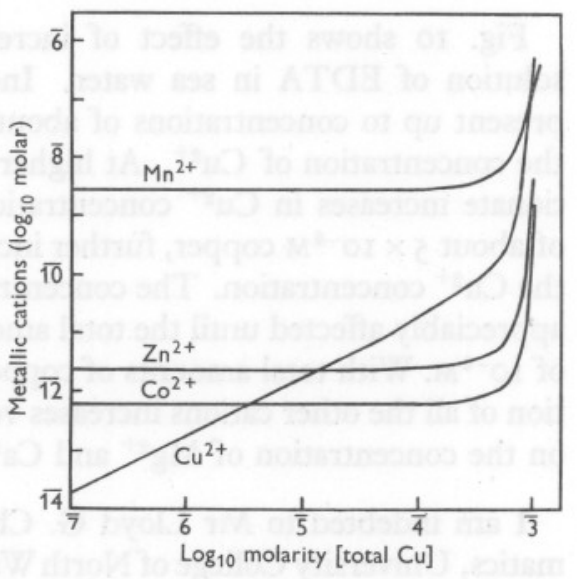

Fig. Io

Fig. 9. Ratio of $f$ at various total calcium concentrations to $f$ at $10^{-2} M$ total calcium in $19 \%$ $\mathrm{Cl}$ sea-water solutions of EDTA at $\mathrm{pH} 8.0$.

Fig. Io. Variation in the concentration of certain metallic cations in $10^{-3} \mathrm{M}$ solutions of EDTA in $19 \% \mathrm{Cl}$ sea water at $\mathrm{pH} 8.0$ with additions of various amounts of copper.

In contrast, if the total concentration of a minor constituent is increased, small increases have no appreciable effect on the value of $f$ and only the concentration of the free cations of the metal in question will increase. When the total concentration of such a minor constituent is increased above a certain critical value, however, the corresponding term in the general formula will become of significance in controlling the value of $f$.

Table 3 gives the values of $f$ appropriate to several total concentrations of some biologically important minor constituents. The concentration at which a particular metal will start to affect the other equilibria and the magnitude of the effect produced depend upon the stability constant of the chelate of the 
metal in question. Thus the other equilibria will be affected sooner by increases in the total amount of copper than by increases in less firmly bound metals such as manganese.

TABLE 3. VARIATIONS OF $f$ IN $10^{-3} \mathrm{M}$ EDTA SOLUTIONS IN $19 \%$ CL SEA WATER AT $\mathrm{pH}$ 8.0 WITH CHANGES IN THE TOTAL AMOUNTS OF CERTAIN BIOLOGICALLY IMPORTANT MINOR CONSTITUENTS

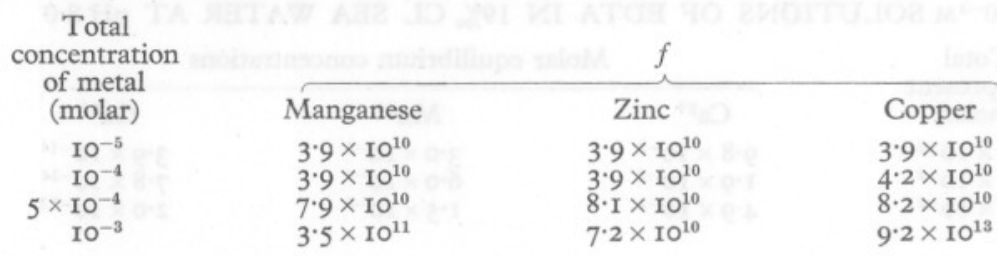

Fig. Io shows the effect of increases in the total copper in a typical solution of EDTA in sea water. Increases in the total amount of copper present up to concentrations of about $1 \mathrm{O}^{-5} \mathrm{M}$ cause proportional increases in the concentration of $\mathrm{Cu}^{2+}$. At higher concentrations increasingly disproportionate increases in $\mathrm{Cu}^{2+}$ concentrations are caused until, at concentrations of about $5 \times 1 \mathrm{IO}^{-4} \mathrm{M}$ copper, further increases result in a very rapid increase in the $\mathrm{Cu}^{2+}$ concentration. The concentration of the other metallic cations is not appreciably affected until the total amount of copper in the system is in excess of $1 \mathrm{IO}^{-4} \mathrm{M}$. With total amounts of copper in excess of $5 \times 1 \mathrm{O}^{-4} \mathrm{M}$ the concentration of all the other cations increases very rapidly. There will be similar effects on the concentration of $\mathrm{Mg}^{2+}$ and $\mathrm{Ca}^{2+}$.

I am indebted to Mr Lloyd G. Chambers of the Department of Mathematics, University College of North Wales, for advice and a valuable discussion.

\section{SUMMARY}

A general formula has been derived for the quantitative treatment of systems involving a number of interdependent equilibria. The formula has been applied specifically to the equilibria set up between metallic cations and their chelates when EDTA is added to sea water. This system involves competition for the fully dissociated quadrivalent anion of EDTA (the principal ion of EDTA which forms complexes) by the metallic cations and by the hydrogen ions present. The equilibrium concentration of a metallic cation in any particular case will depend upon the equilibrium concentration of the quadrivalent anion of EDTA, in the sense that the higher the concentration of this ion the lower will be the concentration of the metallic cation. The general formula allows the calculation of the equilibrium concentration of the quadrivalent EDTA anion, and from this the concentrations at equilibrium of each metallic cation can be readily found. 
When solutions of EDTA in typical sea waters are considered quantitatively, it becomes apparent that relatively few of the metallic cations present contribute significantly to determining the equilibrium concentration of the EDTA quadrivalent anion. The mass action effect of the relatively large concentrations of calcium and magnesium in sea water causes these ions to be dominant in controlling the equilibria. Consequently, changes in the total amounts of either of these molecular species cause marked changes not only in the equilibria in which they are directly involved but also affect the other equilibria. In contrast, changes within certain limits in the total concentration of other metal species have but little effect on the equilibrium concentration of the EDTA quadrivalent anion, and so affect only the equilibrium involving the metal ion in question. The limits within which this applies are controlled by the concentration at which the mass effect of the metal species in question becomes great enough to cause that particular equilibrium to have a significant effect in controlling the concentration of the EDTA quadrivalent anion. The concentration at which this occurs depends in turn upon the magnitude of the stability constant of the metal-EDTA chelate in question. The more stable the chelate, the lower the upper limit of the concentration range over which the total amount of a metal may be altered without appreciably affecting the other equilibria.

Variations in the amount of EDTA present affect all the equilibria. With lower amounts of EDTA (up to about $10^{-4} \mathrm{M}$ ) there is an approximately inverse proportionality between the total amount of EDTA present and the concentration of unchelated metallic cations. As the total amount of EDTA approaches a value stoichiometrically equivalent to the total amount of divalent cations present (i.e. about $6 \times 1 \mathrm{IO}^{-2} \mathrm{M}$ in a typical sea water), there is a progressive disproportionate decrease in the equilibrium concentration of each metallic cation.

An examination of the effects of $\mathrm{pH}$ on the system shows that in sea-water solutions of EDTA, $\mathrm{pH}$ will have little effect on the equilibrium concentrations of free metal ions within the range $\mathrm{pH}$ 6-Io. Below $\mathrm{pH} 6$ increases in the metallic cation concentration will occur. These increases will be proportionately greater in the case of the minor constituents of sea water. The relative $\mathrm{pH}$-independence of the system above $\mathrm{pH} 6$ is limited to systems in which divalent metal cations are in stoichiometric excess of the EDTA.

The equilibria in EDTA-sea water systems are dependent upon the ionic strength of the medium and hence on the salinity. A fall in salinity with its concomitant decrease in ionic strength is associated with increased chelation.

Graphs are presented showing the variations of the reciprocal of the concentration of the quadrivalent EDTA anion $(f)$ with variations in those factors affecting the equilibria which are summarized above. These will allow an approximate evaluation of the ionic concentrations of metallic cations in many cases which may be encountered in biological work. For more precise 
calculations the interpolated values of $f$ may be used as a first approximation to the root of the appropriate equation for $f$, which may then be refined to whatever degree is desired.

The general procedures used are applicable to many biological fluids other than sea water, and for similar systems of competing equilibria other than the formation of complexes.

\section{REFERENCES}

Calvin, M. \& Martell, A. E., 1953. Chemistry of Metal Chelate Compounds. New York: Prentice-Hall, Inc.

Carini, F. F. \& MARTELl, A. E., I952. The effect of potassium chloride on the equilibrium between ethylene diamine tetra-acetate and calcium ions. F. Amer. chem. Soc., Vol. 74, pp. 5745-8.

Chaberek, S., Bersworth, F. C. \& MARTell, A. E., 1955. Chelating agents as metal buffers in biological systems. I. Principles and use of ethylene diamine tetraacetic acid. Arch. Biochem. Biophys., Vol. 55, pp. 321-37.

Mitchell, P. H. \& Rakestraw, N. W., 1933. The buffer capacity of sea water. Biol. Bull., Woods Hole, Vol. 65, pp. 437-5I.

RaAfLaUb, J., 1956. Application of metal buffers and metal indicators in biochemistry. Methods of Biochemical Analysis, Vol. 3, pp. 301-25. New York: Interscience Publishers, Inc.

Sverdrup, H. U., Johnson, M. W. \& Fleming, R. H., 1942. The Oceans, their Physics, Chemistry and General Biology. New York: Prentice-Hall, Inc. 